\title{
CORRECTION
}

\section{Correction: Biomarkers of ageing in New Zealand VLBW young adults and controls}

Brian A. Darlow, John Horwood, Bhubaneswor Dhakal, Sarah L. Harris, Victoria A. McKelvey, John M. Elliott, Jun Yang and Richard J. Mackay

Pediatric Research (2021) 89:708; https://doi.org/10.1038/s41390-020-1054-8

Correction to: Pediatric Research https://doi.org/10.1038/s41390020-0882-x, published online 15 April 2020

The original version of this Article contained an error in the spelling of the author Bhubaneswor Dhakal, which was incorrectly given as Bhubaneswar Dhakal. This has now been corrected in both the PDF and HTML versions of the Article. 